\title{
Radiographic characteristics of appendicular osteosarcoma in a six years old entire Rottweiler Bitch - A case report and a review of the literature
}

\author{
Omamegbe, J. 0. ${ }^{{ }^{*}}$, Adeyemo, B. T. ${ }^{2}$, Okafor, R. S. 0. ${ }^{1}$, Umeakuana, P. U. ${ }^{3}$, Onah, J. \\ A. ${ }^{1}$ and Audu, Z. ${ }^{4}$ \\ ${ }^{1}$ Department of Veterinary Surgery, University of Abuja, Abuja, Nigeria. \\ ${ }^{2}$ Department of Animal health and production, University of Abuja, Abuja, Nigeria. \\ ${ }^{3}$ Department of Veterinary Medicine, University of Abuja, Abuja, Nigeria. \\ ${ }^{4}$ Veterinary Care Clinics, Wuse Zone 5, Abuja, Nigeria. \\ *Corresponding author. Email: josephomamegbe@yahoo.com
}

Copyright (@ 2017 Omamegbe et al. This article remains permanently open access under the terms of the Creative Commons Attribution License 4.0, which permits unrestricted use, distribution, and reproduction in any medium, provided the original work is properly cited.

Received 4th August, .2017; Accepted 28th August, 2017

\begin{abstract}
A six years old entire Rottweiler bitch which was presented with the sole history of mild lameness and mild pain of the right forelimb was diagnosed as appendicular osteosarcoma (APOSA) based on its general condition, the physical and radiographic examinations supported by biochemical findings. The radiographic findings revealed an aggressive osseous growth, a "sunburst" appearance, a Codman's triangle, the presence of a skip metastasis, mixed osteoblastic, osteosclerotic, osteolytic changes, erosion of the cortex of the affected humerus and rapidly growing metastases in the pulmonary system. There were serial elevations of the serum alkaline phosphatase levels during three consecutive occasions when it was assessed from 249 to 307 to $715 \mathrm{IU} / \mathrm{L}$ within eleven weeks as against the normal level of 1.0 to $114.0 \mathrm{IU} / \mathrm{L}$. The anamnesis, clinical, radiographic and biochemical findings indicated a grave prognosis. No specific treatment or management protocol was administered as the client opted for none that was suggested. The bitch was eventually euthanized 11 weeks after its initial presentation due to its rapidly deteriorating health and a grave prognosis based on negatively progressive radiographic indices and a rising serum alkaline phosphatase profile. This presentation details the radiographic features recorded (in probably the first documented case of OSA in Nigeria) in an entire six years old Rottweiler bitch.
\end{abstract}

Keywords: Appendicular, characteristics, Osteosarcoma, radiographic, Rottweiler.

\section{INTRODUCTION}

Osteosarcoma (OSA) is the commonest osseous tumor in dogs. It accounts for about 5 to $6 \%$ of all canine neoplasias and 75 to $80 \%$ of bone tumors in dogs. Up to 70 to $80 \%$ of OSAs affect the appendicular skeleton while 20 to $30 \%$ occur in the axial skeleton (Thrall and Goldschmidt, 1985). Although OSA presents characteristic radiographic changes, its diagnosis based on the signalment, anamnesis, presenting clinical signs and radiological findings alone can be very difficult, particularly in the early stages of its development (Thrall and Goldschmidt, 1985) especially by veterinarians who are not familiar with its subtle and often innocuous clinical manifestations during this stage. The annual occurrence of new cases of OSA in the USA has been estimated at 8 to 10 thousand (Mirabello et al., 2009; Withrow and MacEwen, 1989; Rowell et al., 2011).

The authors of this report are not aware of any previous documentation of OSA among the canine species in Nigeria. The condition seems to be very rare in the country probably because the large to giant dog breeds that are predisposed to it are relatively few among the Nigerian canine population (Omamegbe, 1980; Ajadi et al., 2013). The occurrence of canine OSA is expected to be low in environments/countries where large to giant 
canine breeds like the Alsatians, St. Bernard, Rottweiler, Great Dane, Irish Setter, Doberman Pincher and Golden Retriever are few and especially if only a small proportion of them live up to the middle to old age brackets during which OSA appears to be prevalent in canines (Fenger et al., 2014). Because OSA is seldom seen among canines in Nigeria, Veterinarians in the local settings have seemingly developed a low suspicion index for canine OSA such that the rare cases, that may occur, go unrecognized when presented.

Appendicular osteosarcoma (APOSA) is reported to be more common in the forelimbs which bear more of the body weight than in the hind limbs and at the zones of rapid bone growth on long bones but away from growth plates and the epiphyses. Osteosarcomas, including APOSAs, are composed of varied proportions of osteod, fibroid or chondroid ground tissue with varied degrees of ossification. On account of this, it has been claimed that OSAs appear to develop from pluripotent parent cells very close to, but not from, stem/germinal cells directly.

The typical clinical signs of APOSA in man and canines are slowly developing lameness which starts very mildly but progresses in severity to incapacitation, swelling of the affected limb especially terminally, idiopathic or folding/pathologic fractures and progressive deterioration of the health associated with respiratory distress due to increasing numbers of, bigger and consolidated metastases from the tumor in the lungs.

In recent years, the diagnosis and management of canine OSA has advanced so much that they now approximate practices and treatment modules currently used in human OSA. Advanced thigh technology noninvasive imaging techniques such as magnetic resonance imaging (MRI), computed tomography (CT), and positron emission tomography and recently the $3-D$ radiographic techniques are currently used in the diagnosis of canine OSA. This advancement have been made feasible because of the close similarities of OSA in the two species, several collaborative researches employing OSA in canines as a model in the study of human OSA and more importantly because most of these diagnostic innovations were based on researches using naturally occurring canine OSA (Khanna et al., 2006). Also, the management of canine OSA now includes different modules incorporating various combinations of amputation (total or limb-sparing coupled with chemotherapy - adjuvant and neo adjuvant and radiotherapy) similar to those used in the management of human OSA with comparable outcomes (Liptak et al., 2006; Fenger et al., 2014). Canine OSA is very suited for these research efforts because of the relatively high prevalence of OSA in canines (13.5/100,000.00 canines) as opposed to the prevalence in humans $(1.4 / 100,000.00$ humans), the relatively short time to complete an experimental cycle in canines as opposed to humans, and the ethical, social and cultural considerations involved in experimenting on humans such as serial sampling and end-point assessments.

Hence, the objectives of this report are to alert veterinarians of this seemingly rare condition which may nonetheless be more prevalent than it currently appears to be among the few giant and large dog breeds found in Nigeria and to indicate the role radiography and to some extent serum biochemistry (in particular sAlkphos estimations) could play in the absence of more sophisticated clinical investigatory tools like MRI, CT and PET.

\section{CASE REPORT}

A six years old entire Rottweiler bitch weighing 54.0 kilograms and 34 centimeters high at the shoulders (Figure 1) was referred to the Veterinary Teaching Hospital (VTH), University of Abuja (UA) with the history of mild lameness on the right forelimb which was first noticed about three weeks earlier. The bitch was up-to date on all vaccination programs. It was kept essentially as a pet and guard. The appetite was reported to be normal. Apart from the lameness which at first presentation was adjudged to be mild, no other clinical sign of ill health was reported. There was no history or clinical sign of dyspnoea, coughing or exercise intolerance or of any other systemic dysfunction found on clinical examination. The vital parameters were all normal except for a mild enlargement of the right prescapular lymph node which was only slightly painful on palpation. The bitch exhibited signs of mild pain when the lame (right) forelimb was palpated. The limb felt unusually cold to touch and the humeral region felt enlarged and hard apparently all through its entire length. The body condition of the bitch on the problem - oriented medical record (POMR) Crane, 1990) scale was good.

A routine haematological examination revealed essentially normal values with a slight lymphocytosis and a slight shift to the right. The only clinically significant finding on serum biochemical evaluation was an elevated alkaline phosphatase level (249 IU/L) as against the normal value of 1.0 to $114.0 \mathrm{IU} / \mathrm{L}$ (Boyd, 1984).

A tentative diagnosis of a right forelimb lameness of idiopathic origin was made and a radiographic examination of the affected forelimb region was carried out. This revealed an aggressive exostotoic mass on the surface of the right humerus and a small exostosis on the distal radial metaphysis of the right forelimb. The exostosis on the humerus had permeated into the adjacent soft tissues portraying a "sunburst appearance. This finding suggested a diagnosis of humeral cancer with differentials of mycotic or chronic osteomyelitis. Survey radiographs of the other osseous tissues and the thoracic region showed no growth in any other site of the appendicular or axial skeleton. A lateral radiograph of the thoracic region showed clearly circumscribed, cloudy, radio-opaque lesions widely dispersed in the lungs. These lesions were suspected to be metastases from the primary humeral tumor. 


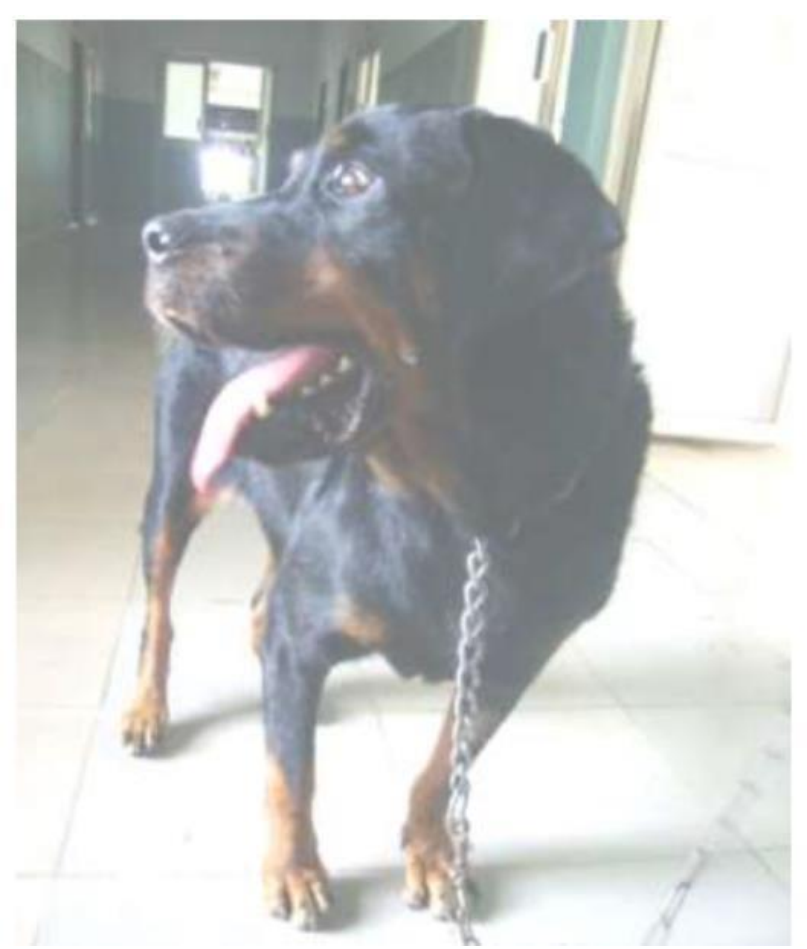

Figure 1. The Patient- A 52 kilograms, $34 \mathrm{~cm}$ shoulder high, six-year old, entire, Rottweiler bitch. Notice that the bitch attempts to shift the weight on the healthy left forelimb. At this stage, the affected right forelimb was cold to touch and mildly painful to moderate palpation but was not swollen.

The definitive diagnosis was osteosarcoma of the appendicular skeleton. The clinical, radiographic and serum biochemical findings suggested a poor prognosis. All through the three occasions when the case waspresented to the hospital, the client did not give permission for euthanasia or amputation of the right forelimb to be followed by chemotherapy as was suggested.

The bitch was re-presented to the clinic three and eleven weeks thereafter. At each of these further presentations, the patient's general health condition had progressively deteriorated. There were in addition progressive elevations of the serum alkaline phosphatase level to $307 \mathrm{IU} / \mathrm{L}$ and $715 \mathrm{IU} / \mathrm{L}$ during the second and third consultations.

Serial radiographs of the appendicular and axial skeletons and the thoracic cavity taken on these two occasions showed that the metastases in the lungs were progressively getting bigger in size and more consolidated.

The bitch was eventually euthanized because of the deteriorating clinical condition, the progressive increases in the sizes of the primary humeral tumor and the lung metastases and the evidence of continually rising levels of serum alkaline phosphatase - all indicative of a very grave prognosis.

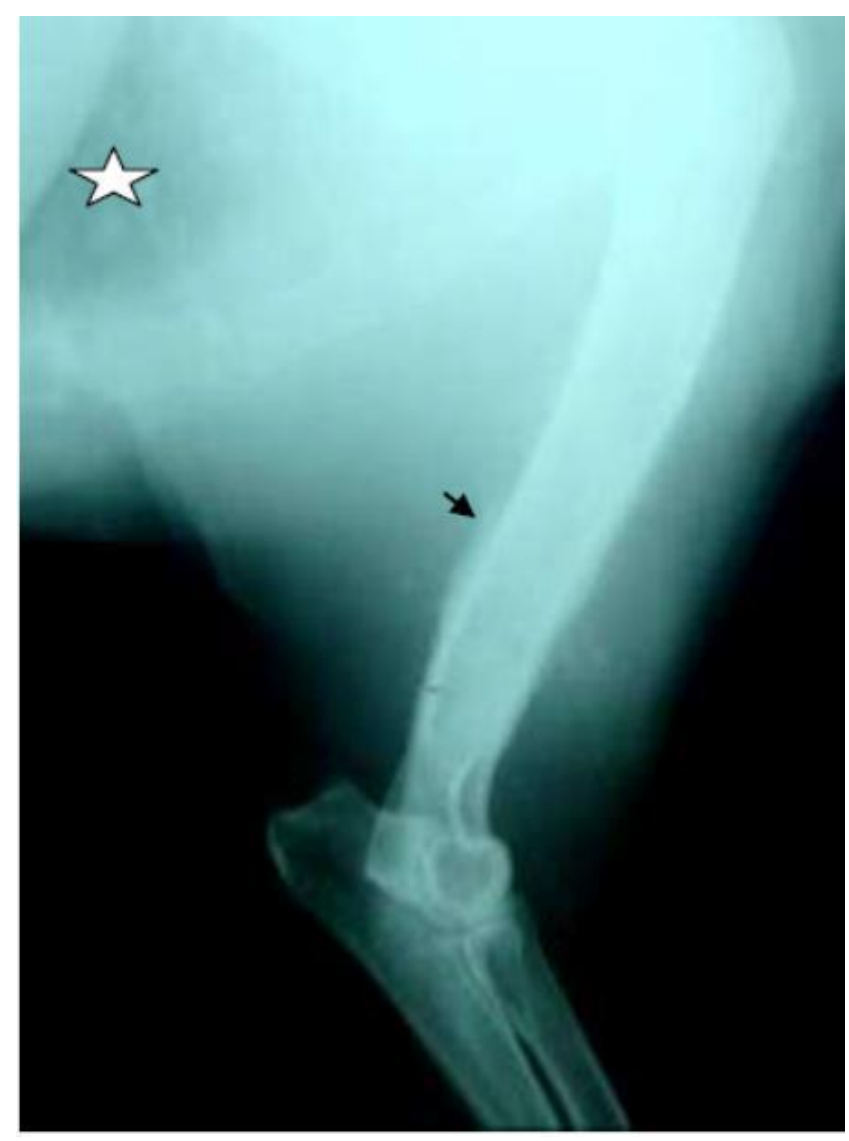

Figure 2. Radiology of the right humerus. Notice the exostosis/periosteal reaction permeating into surrounding soft tissues giving a "sunburst" appearance, mottled appearance of the medullary cavity of the humerus resulting in the loss of the spicules in the metaphysis, non-involvement of the epiphysis, the elbow joint and the adjacent proximal radius and ulna bones. The cortex of the humerus at the epiphyseal region is essentially normal. A "Codman's triangle" was seen (arrow). Notice metastases of tumor in the cardiac lung lobe (star).

\section{Solitary location of the tumor}

The lesion was limited essentially to the metaphyseal and diaphyseal regions of the right humerus and a small exostosis on the cranial aspect of the distal radial metaphysis of the right forelimb (Figure 2). No tumor growth was found at any other site. According to Thrall and Goldschmidt (1985) and Wittig (2014), APOSA is normally found on the metaphysis or the diaphysis or both of one long bone except if the tumor has metastasized to other locations on the same or other bones or body tissues/organs. In such cases, the metastases are smaller than the primary growth. Metastases to the same or other long bones are regarded as skip metastasis and their presence does not distract from the monostotic nature of APOSA. The metastasis found in the distal radial metaphyseal region is considered a synchronous skip metastasis (Figure 3). 


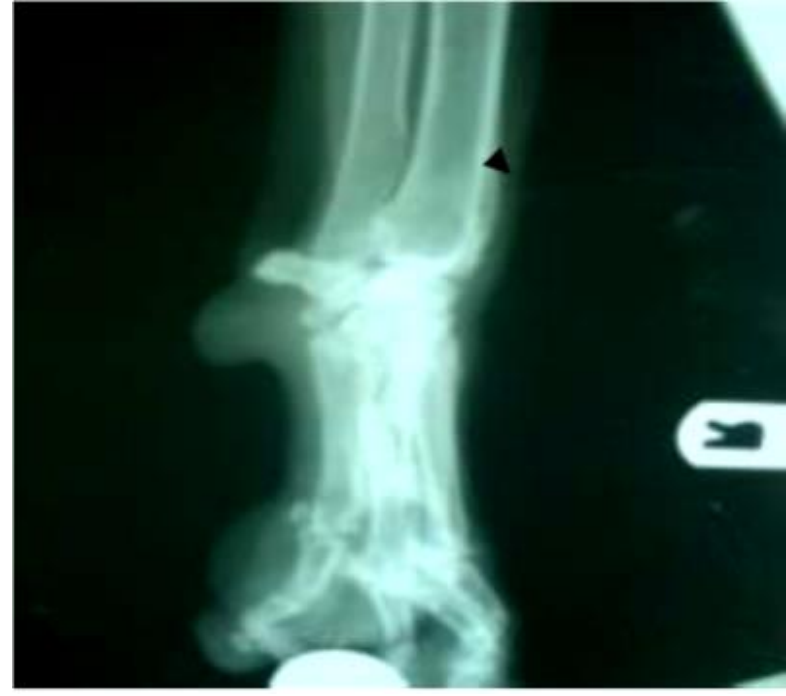

Figure 3. Notice a small growth at the distal epiphyseal region of the right radius (solid arrow head). This is a suspected synchronous skip metastasis. It is much smaller than the primary growth.

\section{Aggressive or permeative nature}

The radiograph of the affected limb showed a wide spread invasion of the surrounding soft tissues which gave a typical "sunburst" appearance - an evidence of metastasis. This and the Codman's triangle (Figure 2) are frequent radiographic findings in, but are however not pthognomonic for, canine APOSA as they could be found in other conditions such as old healing fractures, mycotic osteomas etc (Thrall and Goldschmidt, 1985). There was also blurring of the margin between the cortex of the humerus and the growth in the soft tissues. The cortices of the humerus have been largely destroyed. These features depict the aggressive or permeative nature of the growth (Figure 4).

\section{Nature of the tumor growth}

The primary tumor and skip metastasis exhibited essentially osteoblastic and osteosclerotic characteristics radiographically. Terminally however, there was some osteolytic reaction in the mid-humeral region of the affected humerus as depicted by some increased radiolucency in that region of the humerus between the black arrows (Figure 5). This dog would have possibly suffered from pathological/folding fractures if it was exerted or stressed.

\section{Limitation of the primary lesion}

At the initial presentation, the primary growth was limited to the diaphyseal and metaphyseal regions of the right humerus while the proximal and distal epiphyses were

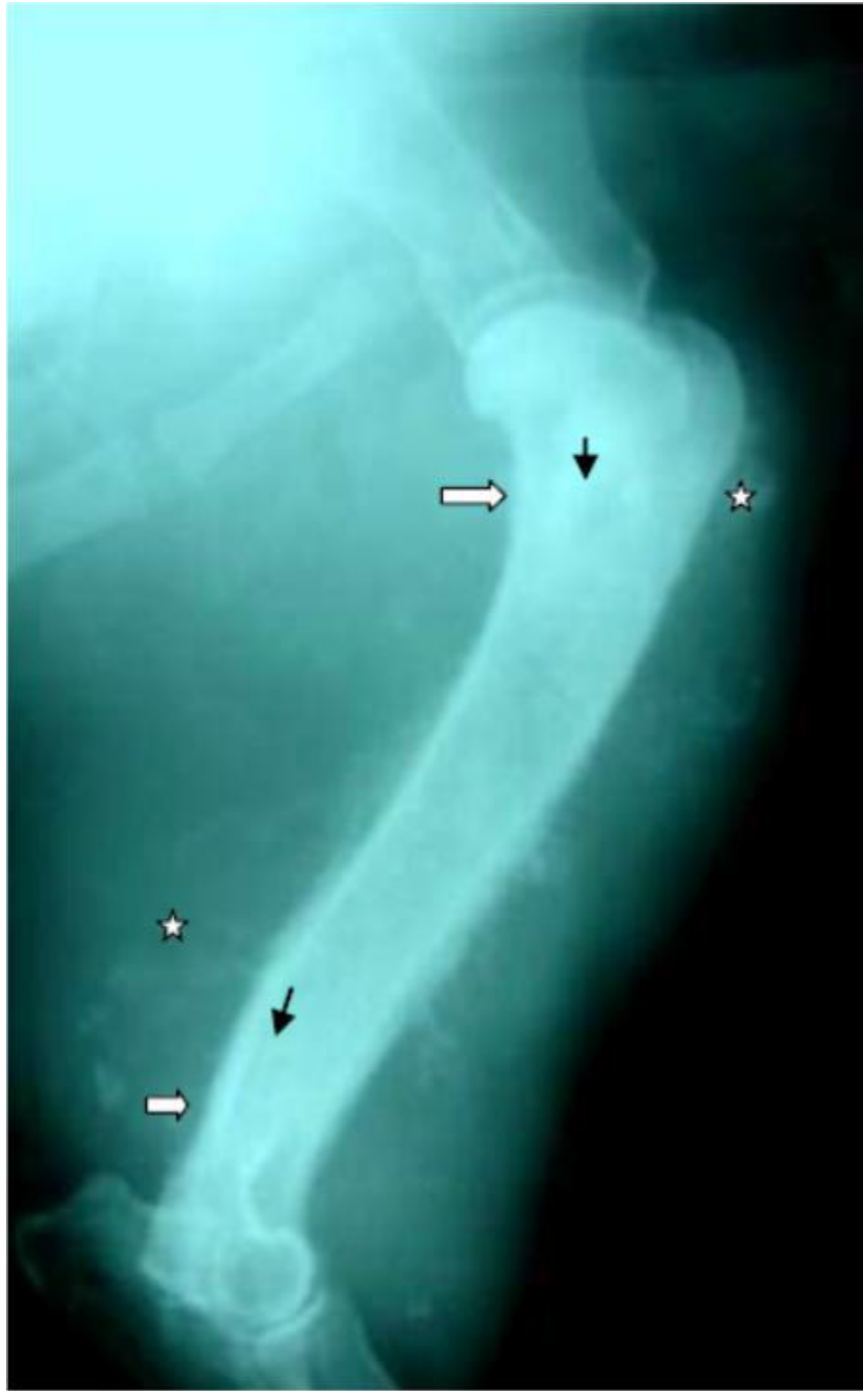

Figure 4. Radiograph of the affected right humerus three weeks later. Physically, the mass had become much bigger on palpation. The ossification of the soft tissues around the humerus has become more evident radiographically giving a more pronounced "sunburst" appearance. The periosteal reaction extends to both the metaphysis and the diaphysis of the humerus (open arrows). The epiphyses and articular surfaces are spared. There are widely dispersed areas of osteosclerosis (stars). Notice the lytic mid humeral region (more radioluscency) and mottling of the medullary cavity (black arrows).

relatively free of tumor growths (Figure 2). But on presentation terminally, the proximal and distal metaphyses and epiphyses had been invaded by tumor growths (Figure 5). The linear growth of APOSA is thought to be limited by open growth plates in young canines. However, in adult canines where the growth plates have closed, APOSA could progress across the fused growth plates and be prevented from invading the joint and the adjacent bone by the articular cartilage only (Thrall and Goldschmidt, 1985). 


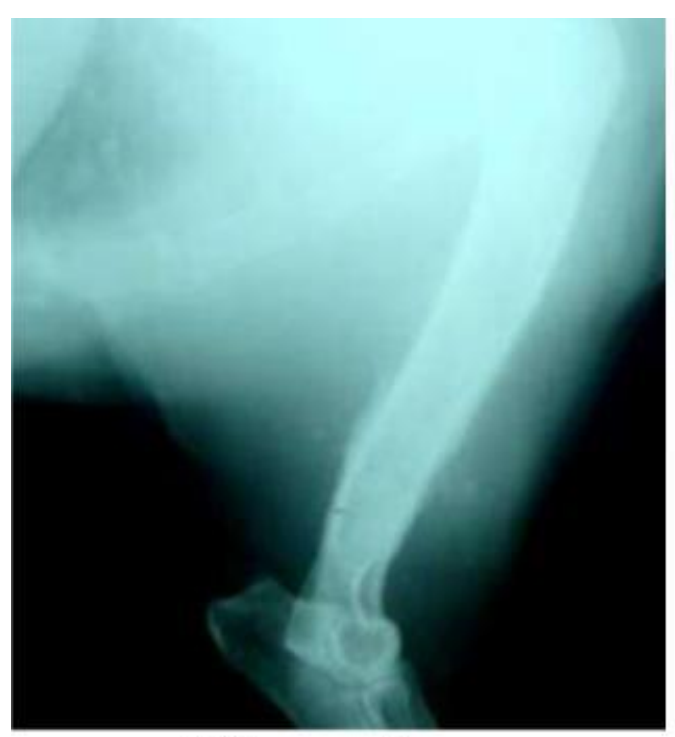

At three weeks

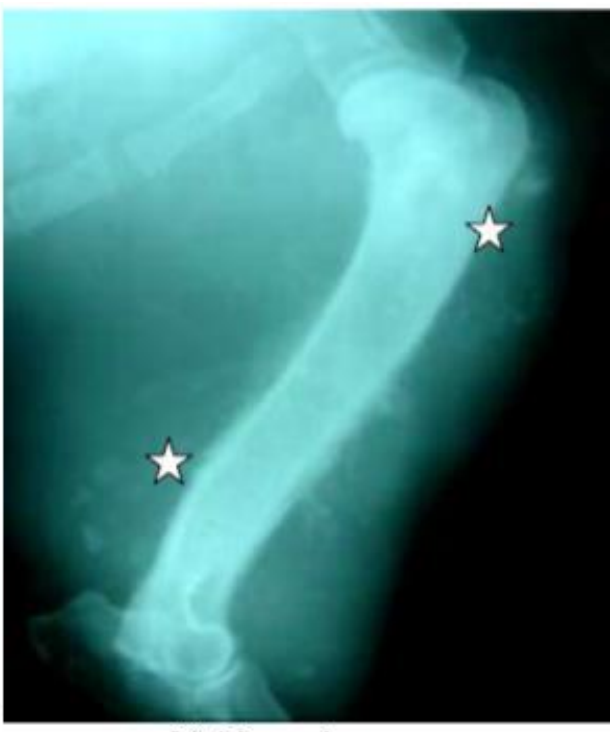

At 14 weeks

Figure 5.Comparism of the size of the tumor at weeks 3 and 14. At week 14, tumor permeation is more extensive, the epiphyses are invaded and the metastasis into the surrounding soft tissues show widely disseminated/dispersed foci of radio-densities suggestive of calcification/ossification (stars) which are either less or not present at week 3.

\section{Metastatic spread}

The widely disseminated masses in the lungs at the presentation of the case were metastases from the humeral tumor. All malignant tumors metastasize to various body tissues and/organs either haematogenoulsy or through the lymphatic system. Osteosarcomas tend to metastasize more haematogenously than through the lymphatic system. Metastases to the lymph nodes portend grave prognoses irrespective of the treatment modules employed (Fenger et al., 2014; Hillers et al., 2005). It was not ascertained if the slight right prescapular lyphadenomegaly was due to tumor metastasis to the lymph node. Osteosarcomas may spread to any body organ but a majority of such spread is usually to the lungs. Pulmonary metastases are actually present in about $90 \%$ of all cases of APOSA although they can only be demonstrated radiographically in 15 to $25 \%$ of such cases at the time of diagnosis (Gorlick and Khanna, 2010). These pulmonary metastases are often resistant to most cytotoxic chemotherapeutic agents currently in use in the management of canine APOSA. This probably accounts for the very high mortality rates recorded in canine APOSA when compared with APOSA in humans subjected to similar management protocols (Fenger et al., 2014).

The presence of metastases (from the primary tumor) in the lung fields at the initial presentation of the case (Figure 6), suggested that the condition had existed much longer than the case history suggested (three weeks) or that the tumor was hyper aggressive or permeative. The later view would appear to be the case in view of the rapid increase in the size of the metastases in the lungs at 14 weeks. Eleven (11) weeks later (Figure 7), the progressive deterioration of the health of the bitch (Figure 8) and the steady rise in the levels of serum alkaline phosphates from $249 \mathrm{IU} / \mathrm{L}$ to $307 \mathrm{IU} / \mathrm{L}$ and $715 \mathrm{IU} / \mathrm{L}$ during the three occasions it was evaluated.

\section{Comparism of the size of the tumor at weeks 3 and 14}

The tumor permeated the peri-humeral tissues very aggressively and extensively resulting in a massive increase in the physical size of the humeral region in 11 weeks (between the third and fourteenth weeks). The "sunburst" appearance on X-rays is more prominent in Figure 5 than Figure 2. And there are widely disseminated sclerotic foci in the peri-humeral soft tissues (stars in Figure 5). These are signs of permeative/aggressive growth.

Currently, alterations in the serum alkaline phosphatase levels were used as prognostic indicators in canine APOSA intended for or undergoing therapy. High alkaline phosphates levels suggest a poor prognosis particularly if the level continues to rise or remains static after the initiation of therapy (Thorpe et al., 1979; Bicci et al., 1987; Bicci,et al., 1993; Boernan et al., 2012). The steady elevations of the serum alkaline phosphatase level in this case correlated closely with the steady increase in the size of the tumor on the humerus and the lung fields on one hand and with the deterioration in the general health of the bitch on the other (Figure 8). 


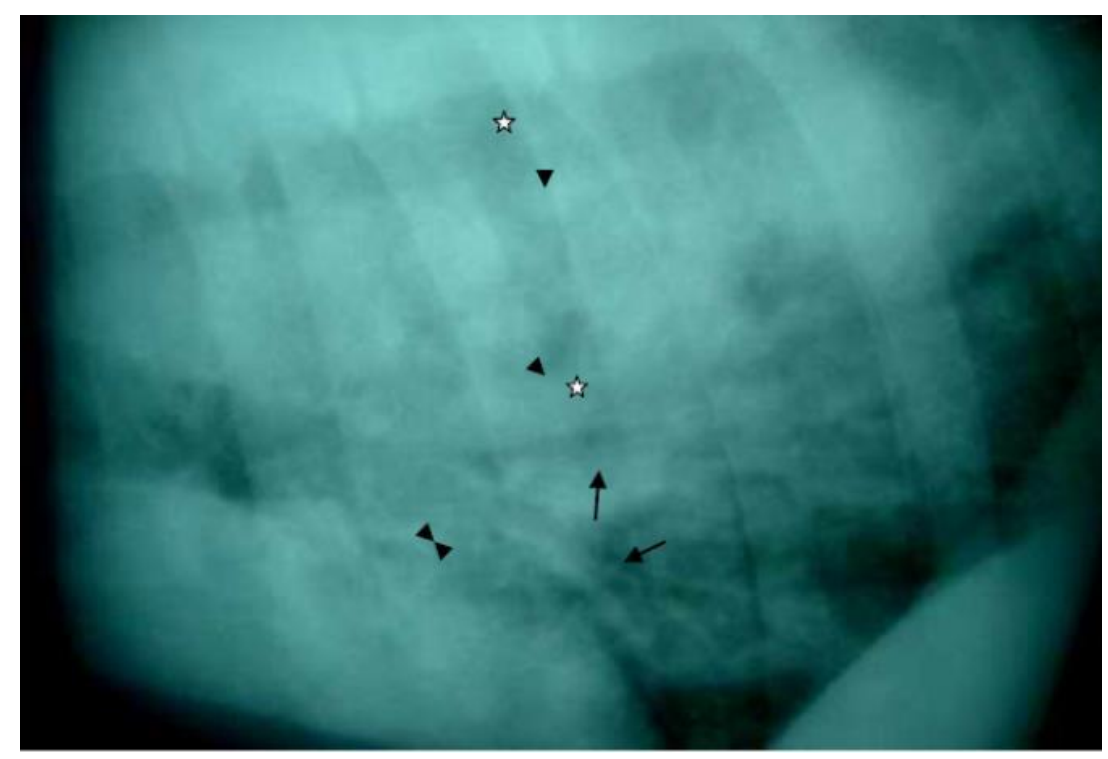

Figure 6. A left lateral radiograph of the thoracic cavity. Notice the increased radio-opacity of the lung fields, lumpy neoplastic growths in all lung lobes (arrow heads) and prominent air-bronchiograms (arrows) and angio/venograms (stars), numerous metastatic disseminated foci of the tumor. Normally, only micro-metastases that are up to 6-8 millimeters in diameter are detectable on thoracic radiographs of affected patients.

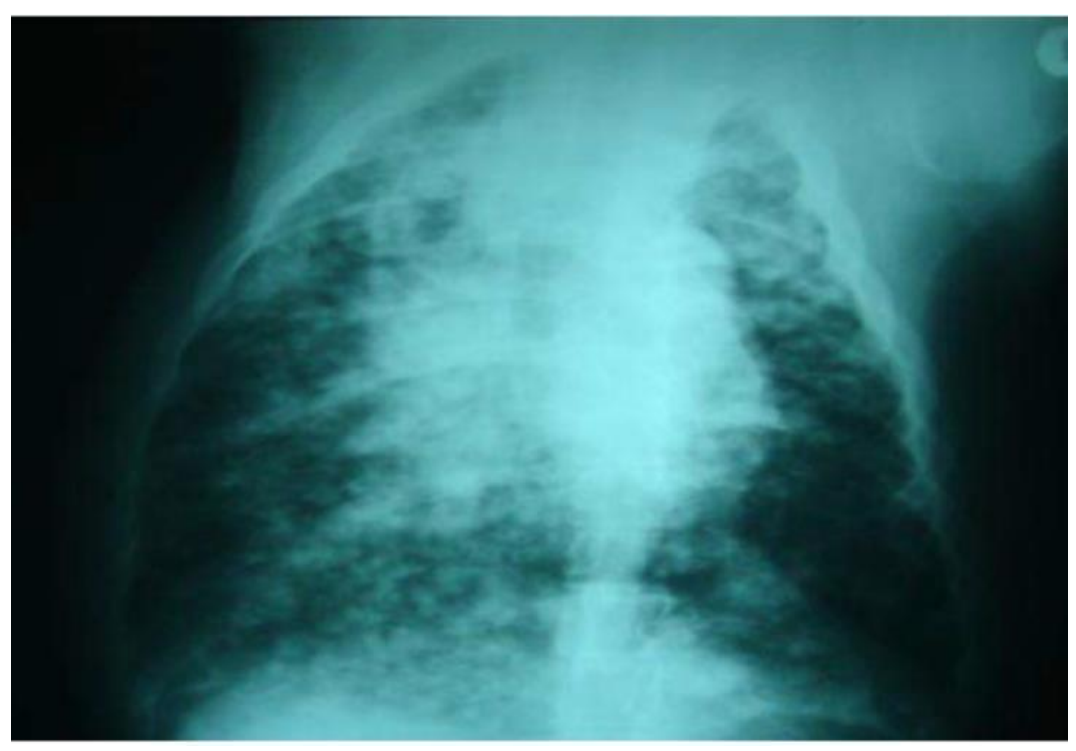

Figure 7. A dorso- ventral radiograph of the thoracic cavity of the bitch terminally. The metastases to the lungs have become more advanced, bigger and coalescing. The lumps of tumor masses have developed clearer margins than at 3 weeks but are not ossified/calcified. They are more disseminated affecting the diaphragm and possibly the liver. The shadows of the heart, the sternum and vertebrae are hardly discernable.

\section{DISCUSSION}

The signalment, anamnesis and the clinical findings in the case reported all agree with those associated with
APOSA in canines and to some extent in humans in both of which APOSA seems to exhibit very profound similarities (Fenger et al., 2014). Of particular attention is the mild and often negligible clinical signs recorded 


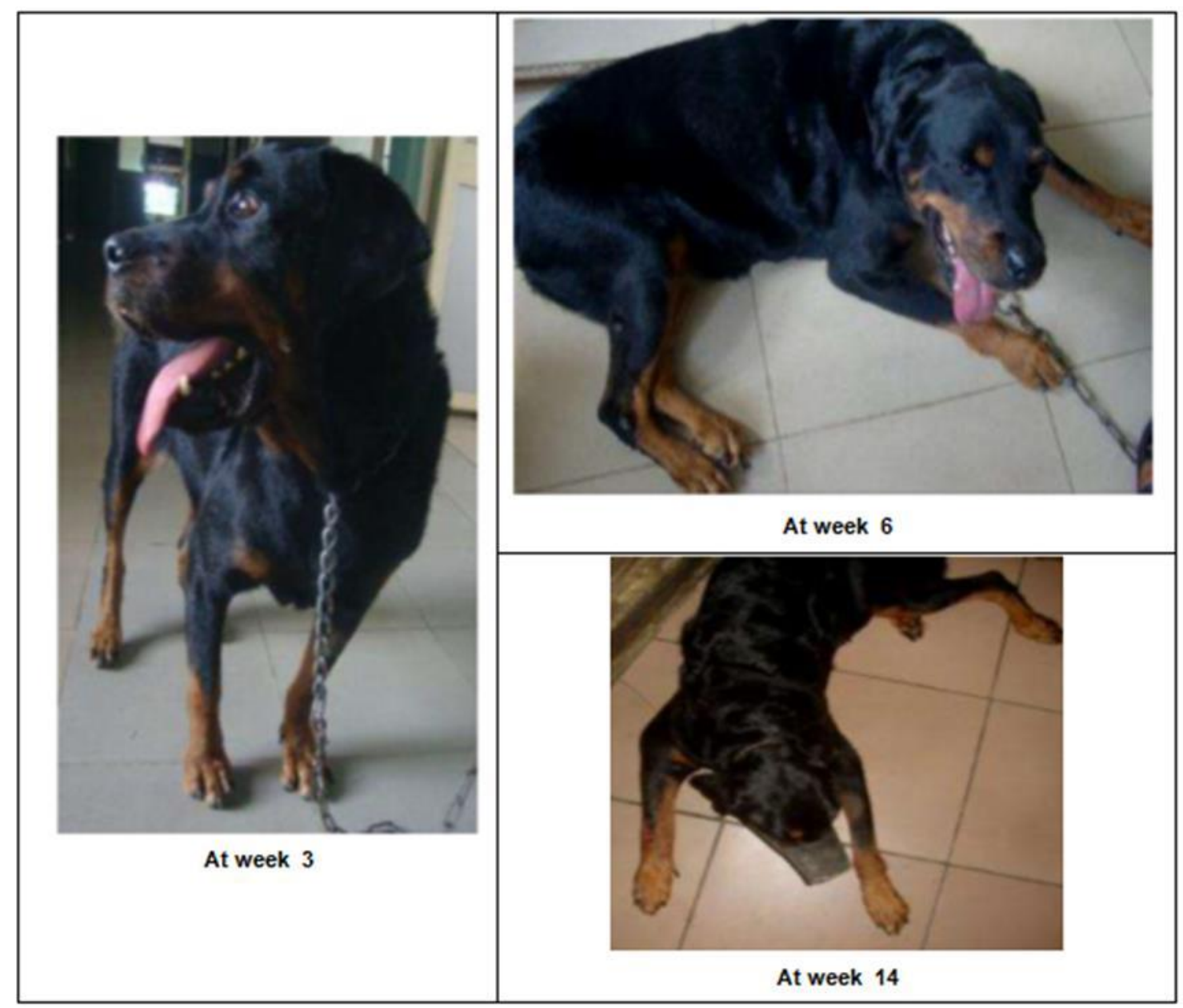

Figure 8. Showing the progressive deterioration of the general health condition from weeks 3 to 6 and 14 (an 11 weeks interval). The entire right forelimb has become obviously swollen at 14 weeks.

during the initial presentation of the case - a phenomenon often encountered in the early stages of the development of APOSA in canines and humans (Gail et al., 2010; Szewczyk et al., 2015). Appendicular osteosarcoma may be suspected in large to giant canine breeds, of the middle age bracket, weighing 45.0 kilogram and above and of high shoulders (35 $\mathrm{cm}$ and above) when they present clinical signs akin to those reported in this case. It has been claimed that the weight and the height at the shoulders are the most reliably predictive parameters for the occurrence of APOSA in canines (Ru et al., 1998; Fenger et al., 2014). The presence of a large palpable mass on a long bone of such a canine, of similar bio-data as above should arouse a strong suspicion of APOSA as it did in the case reported although some other conditions that present similar features should be borne in mind. The most common of such conditions are osseous reaction to old metallic implants, old healing fractures, mycotic infections, hypertrophic osteodystrophy/osteopathy and other bone cancers (Lenehan and Fetters, 1985a, b). The case presented was diagnosed as APOSA rather than any of the above conditions based of the case history, age of the bitch, the monostotic nature of the growth and other radiographic findings (Thrall and Goldschmidt, 1985; Ghadiri et al., 2007; Gail et al., 2010).

The high level of serum alkaline phosphatase (sAlkphos) at the initial presentation of the case corroborated the clinical findings and indicated a rapid cellular turn over only as this enzyme has been regarded as ubiquitous (Boyd, 1984). Alterations in its levels are therefore aspecific for any clinical condition or pathology in any body system including osseous tissues (Corathers, 2006). It could have been necessary to determine the level of the bone specific isomer of the enzyme (BsAlkphos) and the level of serum Lactate dehydrogenase (LDH) for the value of sAlkPhos found in this bitch to be of some reasonable diagnostic value (Chen et al., 2014). These were not determined in the case reported because of the cost implications. However, alterations in the level of sAlkphos had been used as a prognostic parameter in patients with APOSA undergoing 
adjuvant and/or neo-adjuvant therapy in combination with amputation in canines (Boerman et al., 2012;) and in humans (Thorpe et al.,1979; Bacci et al., 1987; Bacci et al., 1993). Its continuously rising level in this case was used only in monitoring the progression of the disease since no specific treatment was given. It also informed its poor prognosis.

The radiographic appearance at the initial presentation of the case showed radiographic characteristics of osteoblastic and osteosclerotic APOSA as described by (Withrow and MacEwen, 1989; Konde, 2002; Cavalcanti et al., 2004; Gail et al., 2010) which later developed into some osteolytic lesions giving a final radiographic expression of a heterogenous APOSA (Gail et al., 2010). It could not be determined if this was a $100 \%$ osteosarcoma or a mixture with fibrosarcoma and/or chondrosarcoma because no histopathological examination was carried out on samples from the tumor. This is absolutely necessary to enable a comprehensive categorization or classification of the tumor (Thrall and Goldschmidt, 1985; Gail et al., 2010) and a more conclusive diagnosis. The restriction of APOSA to the meta- and dia-physes of long bones by the open growth plates is probably because APOSA develops from pluripotent cells located between stem cells found in the growth plates and fully differentiated osseous, fibrous or cartilaginous cells. Thus, the parent cell for OSA are primordial cells more developed or advanced than stem cells but less differentiated than osteoblasts, chondroblasts or fibroblasts. Apparently, the zone at which the mutations which give rise to OSA cells occur is far removed from the growth plates - stem cells zone therefore osteosarcoma can not develop directly at the growth plates (Riser, 1972). This may explain why OSA does not invade the growth plates when they are open but does when they are closed.

The permeative nature of the tumor was evident from the extensive invasion of the surrounding soft tissue, the wooly nature of the exostosis, destruction or thinning of the cortexes of the humerus and the indistinct boarders between the parent bone, the tumor growth and the adjacent soft tissues. These features are regularly associated with APOSAs and are characteristics generally of aggressive or permeative malignant neoplasias (Thrall and Goldschmidt, 1985).

The "sunburst" appearance and the Codman's triangle have been reported by others in APOSA. However, while both are characteristic findings and are highly suggestive of APOSA, they are not pathognomonic for it in canines or humans (Gorlick and Khana, 2010; Ehrhart et al., 2013). Both can be found in chronic inflammatory osseous lesions such as healing fractures, in osseous tissues with long standing metallic implants and mycotic osteomyelitis (Fenger et al., 2014). None of the above conditions was reported or found during the clinical examination of this case. It is however important to determine whether any of these conditions is present in cases similar to that reported here from the point of view of treatment and prognosis. The case was easily distinguished from hypertrophic oesteodystrophy (HOD) because unlike APOSA, HOD occurs in canines less than one year old, is polystotic in nature and induces a radiographically transluscent line at the metaphysical side of the open growth plate of long bones (Ghadiri et al., 2007, Lenehan and Fetter, 1985a, b; Konde, 2002).

The presence of pulmonary and skip metastases at the initial presentation of this case patented a grave prognosis as they together with lymph node metastasis (Hillers et al., 2005) are the main causes of mortality in canine APOSAs even after adjuvant and neo-adjuvant chemotherapies are administered in conjunction with amputation (Wittig, 2014; Ehrhart et al., 2013; Fenger et al., 2014). It is suspected that the tumor may have metastasized to the right prescapular lymph node which was swollen and painful at the initial presentation of the case. This observation could have been ascertained if a lymph node biopsy or needle aspirant was examined. The rapid increase in the size of the primary tumor on the humerus, the metastases in the lungs and possibly the lymph node; and the progression of the osseous pathology to osteolysis all agree with the grade IIIB status given to the tumor (Withrow et al., 1989; Szewczyk et al., 2015). Canine cases of APOSA grade IIIB die within 6 months of their diagnosis with or without treatment (Szewczyk et al., 2015) as occurred in this case.

The rapid deterioration of the health and death of this bitch within 11 weeks of diagnosis agrees with the diagnosis, progression, steadily deteriorating sAlkphos profile and the end point of canine APOSA especially when no specific treatment was administered.

\section{Conclusion}

Cases like the one reported, in which there are demonstrable pulmonary, skip and/or lymph node metastases, should be euthanized rather than subjected to any module(s) of management like amputation cum chemotherapy except if the client objects to euthanasia. However, the cost of amputation with adjuvant and neoadjuvant therapy is prohibitive and is in the region of seven hundred thousand $(700,000.00)$ Nigerian naira. It is also very stressful on the patient, requires a protracted and rigid regimen under specialist veterinary oncologists and is credited with very poor outcomes in humans and canines (Fenger et al., 2014). It may therefore not be a favoured mode of management.

Presently, several conditions in animals, some naturally occurring such as canine APOSA, provide unequalled research resources for the study of various aspects of similar conditions (in this case APOSA) in humans. The outcomes of such research efforts are of immense benefits to humans and animals. Therefore, there is an urgent need for some vigorous public enlightenment 
about the roles animal models can play in researches into conditions that are common to humans and animals generally, and APOSA in particular, to the benefits of both species (Fenger et al., 2014). This is particularly important because in humans experimentation is impossible or impracticable for ethical, socio-cultural and/or safety reasons (Gordon et al., 2009; Khanna et al., 2006; Paoloni and Khanna, 2008). In the authors' view, there is an urgent need for more public enlightenment about this aspect of veterinary contributions to the "One World One Health" slogan in third world/developing countries.

\section{CONFLICT OF INTEREST}

The authors declare that they have no conflict of interest.

\section{REFERENCES}

Ajadi, A., Adeniyi, A. A., Abiade, A., Nogttige, H. O., Otesile., \& Kasali, O. B. (2013). Radiographic diagnosis of skeletal diseases among dogs in Lagos and Ogun States, Nigeria. A preliminary report. Trop. Vet. J., 31, 65-73.

Bacci, G., Pacci, P. M., Orlandi, M., Avella, M., Manfrini, M., Pignitti, G., Dallari, D., \& Manduchi, R. (1987). Prognostic significance of serum alkaline phosphatase in osteosarcoma. Tumori, 73(4), 331-336.

Bacci, G., Pacci, P., Ferrari, S. M., Orlando, M., Ruggieri, P., Casadei, R., Ferraro, A., Biagini, R., \& Battistini, A. (1993). Prognostic significance of serum alkaline phosphatase measurements in patients with osteosarcoma treated with adjuvant and neo-adjuvant chemotherapy. Cancer, 71(4), 1224-1230.

Boerman, I., Selvarajah, G. T., Nielen, M., \& Kirpensteijn, J. (2012). Prognostic factors in canine appendicular osteosarcoma-a meta-analysis. BMC veterinary research, $8(1), 56$.

Boyd, J. W. (1984). Interpretation of serum biochemistry test results in domestic animals. Vet. Clin. Path., 13(2), 7-14.

Cavalcanti, J. N., Amstalden, E. M. I., Guerra, J. L., \& Magna, L. C. (2004). Osteosarcoma in dogs: clinical-morphological study and prognostic correlation. Brazilian Journal of Veterinary Research and Animal Science, 41(5), 299-305.

Chen, J., Sun, M. X., Hua, Y. Q., \& Cai, Z. D. (2014). Prognostic significance of serum lactate dehydrogenase level in osteosarcoma: a meta-analysis. Journal of cancer research and clinical oncology, 140(7), 1205-1210.

Corathers, S. D. (2006). Focus on diagnosis: The alkaline phosphatase level: Nuances of a familiar Test. Pediatrics in Review. 27(10), 382-384.

Crane, S. W. (1990). Principles of Companion Animal Surgery. In: Harvey, C. E., Newton, C. D. \& Schwartz, A (ED). Small Animal Surgery. J. B. Lippincott Co. Philadelphia. Pp. 1-34.

Ehrhart, N. P., Ryan, S. D., \& Fan, T. M. (2013). Tumors of the skeletal syatem. In: Withrow, S. J., Vail, D. M., \& Page, R. L., eds. Withrow and Machwen's Small Animal Clinical Oncology $5^{\text {th }}$ Eds. Loius, M. I. Saundrers Elsevier, Pp. 463-503.

Fenger, J. M., London, C. A., \& Kisseberth, W. C. (2014). Canine osteosarcoma: a naturally occurring disease to inform pediatric oncology. ILAR Journal, 55(1), 69-85.
Gail, Y. M. D., Michael, J., Klein, M. D., Jonathan, L. D. O., Robert, A., Lefkowitz, M. D., \& Sinchum, H. M. D. (2010). Imaging characteristics of primary osteosarcoma: Nonconventional subtypes. Radiographics, 30(6), 1653-1672.

Ghadiri, A. R., Avizeh, R., \& Veshkini, A. (2007). Radiographic findings of hypertrophic osteodystrophy in a mongrel puppy. Iranian Journal of Veterinary Research, 8(2), 178-181.

Gordon, I., Paoloni, M., Mazcko, C., \& Khanna, C. (2009). The Comparative Oncology Trials Consortium: using spontaneously occurring cancers in dogs to inform the cancer drug development pathway. PLoS medicine, 6(10), e1000161.

Gorlick, R., \& Khanna, C. (2010). Osteosarcoma. Bone Miner Research, 25, 683-691.

Hillers, K. R., Dernell, W. S., Lafferty, M. H., Withrow, S. J., \& Lana, S. E. (2005). Incidence and prognostic importance of lymph node metastases in dogs with appendicular osteosarcoma: 228 cases (1986-2003). Journal of the American Veterinary Medical Association, 226(8), 1364-1367.

Khanna, C., Lindblad, - Tok, K., Vail, D., London, C., Hergman, P., Barber, I., Bren, M., Kitchell, B., McNeil, E., Modiano, H., Niemi, N., Comstock, K. T., Ostrander,E., Westmoreland, S., \& Withrow, S. (2006). The dog as a cancer model. Nature Biotechnonoly, 24, 1065-1066.

Konde, I. J. (2002). Diseases of the immature skeleton. In: Thrall, D. E. (ED). Textbook of Veterinary Diagnostic Radiology (4 ${ }^{\text {th }}$ Edition). W. B. Saunders Co. Philadelphia, Pp. 131-142.

Lenehan, T. M., \& Fetter, A. W. (1985a). Hypertrophic osteodystrophy. In: Newton, C. D., \& Nunamaker, D, M. (Eds). Textbook of Small Animal Orthepaedics (Ist eds) J. B. Lippincott Company. Pp. 597-601.

Lenehan, T. M., \& Fetter, A. W. (1985b). Hypertrophic osteopathy In: Newton, C. D., \& Nunamaker, D, M. (Eds). Textbook of Small Animal Orthepaedics (Ist Eds) J. B. Lippincott Company. Pp. 603-609.

Liptak, J. M., Dernell, W. S., Ehrhart, N., Lafferty, M. H., Monteith, G. J., \& Withrow, S. J. (2006). Cortical allograft and endoprosthesis for limb-sparing surgery in dogs with distal radial osteosarcoma: A prospective clinical comparison of two different limb-sparing techniques. Veterinary Surgery, 35(6), 518-533.

Mirabello, L., Troisi, R. J., \& Savage, S. A., (2009). Osteosarcoma - incidence and survival rates from 1973 to 2004. Data from the surveillance, epidemiology and end results programme. Cancer, 115, 1531-1543.

Omamegbe, J. O. (1980). A survey of dogs and their owners seen at two veterinary clinics in the Enugu and Nsukka areas of Anambra State, Nigeria. Nigerian Veterinary Journal, 9, 1016.

Paoloni, M., \& Khanna, C. (2008). Translation of new cancer treatments from pet dogs to humans. Nature reviews. Cancer, 8(2), 147.

Riser, W. H., Brodey, R. S., \& Biery, D. N. (1972). Bone infarctions associated with malignant bone tumors in dogs. J. Am. Vet. Med. Assoc., 160, 414-421.

Rowell, J. L., McCarthy, D. O., \& Alvarez, C. E. (2011). Dog models of naturally occurring cancer. Trends in molecular medicine, 17(7), 380-388.

Ru, G., Terracini, B., \& Glickman, L. T. (1998). Host related risk factors for canine osteosarcoma. The Veterinary Journal, 156(1), 31-39.

Szewczyk, M., Lechowski, R., \& Zabielska, K. (2015). What do we know about canine osteosarcoma treatment? - A review. 
Veterinary research communications, 39(1), 61-67.

Thorpe, W. P., Reilly, J. J., \& Rosenberg, S. A. (1979). Prognostic significance of serum alkaline phosphatase measurements in patients with osteosarcoma receiving chemotherapy. Cancer, 43(6), 2178-2181.

Thrall, D., \& Goldschmidt, M. H. (1985). Radiography and biopsy of bony neoplasia. In: Newton, C. D. and Nunamaker, D, M. (Eds). Textbook of Small Animal Orthepaedics (Ist Eds) J. B. Lippincott Company.
Withrow, S. J., \& MacEwen E. G. (1989). Tumors of the skeleton. In: Clinical Veterinary Oncology Philadelphia. Pa: J B Lippincott Co. Pp. 378 -392.

Wittig, J. (2014). Osteosarcima (Conventional): Bone Tumor Cancer. www.tumorsurgery.org. 